\title{
Fringe Projection Techniques: Whither we are?
}

\author{
Sai Siva Gorthi and Pramod Rastogi \\ Applied computing and mechanics laboratory, Swiss Federal Institute of Technology, 1015 Lausanne, Switzerland.
}

During recent years, the use of fringe projection techniques for generating three-dimensional (3D) surface information has become one of the most active research areas in optical metrology. Its applications range from measuring the 3D shape of MEMS components to the measurement of flatness of large panels $(2.5 m \times .45 \mathrm{~m})$. The technique has found various applications in diverse fields: biomedical applications such as 3D intra-oral dental measurements [1], non-invasive 3D imaging and monitoring of vascular wall deformations [2], human body shape measurement for shape guided radiotherapy treatment [3, 4], lower back deformation measurement [5], detection and monitoring of scoliosis [6], inspection of wounds [7, 8] and skin topography measurement for use in cosmetology [9, 10, 11]; industrial and scientific applications such as characterization of MEMS components [12, 13], vibration analysis $[14,15]$, refractometry [16], global measurement of free surface deformations [17, 18], local wall thickness measurement of forced sheet metals [19], corrosion analysis [20, 21], measurement of surface roughness [22, 23], reverse engineering $[24,25,26]$, quality control of printed circuit board manufacturing [27, 28, 29] and heat-flow visualization [30]; kinematics applications such as measuring the shape and position of a moving object/creature [31, 32] and the study of kinematical parameters of dragonfly in free flight [33, 34]; biometric identification applications such as 3D face reconstruction for the development of robust face recognition systems [35, 36]; cultural heritage and preservation [37, 38, 39] etc.

One of the outstanding features of some of the fringe projection techniques is their ability to provide high-resolution, whole-field 3D reconstruction of objects in a non-contact manner at video frame rates. This feature has backed the technique to pervade new areas of applications such as security systems, gaming and virtual reality. To gain insights into the series of contributions that have helped in unfolding the technique to acquire this feature, the reader is referred to the review articles in this special issue by Song Zhang, and Xianyu Su et al.

A typical fringe projection profilometry system is shown in Fig 1. It consists of a projection unit, an image acquisition unit and a processing/analysis unit. Measurement of shape through fringe projection techniques involves (1) projecting a structured pattern (usually a sinusoidal fringe pattern) onto the object surface, (2) recording the image of the fringe pattern that is phase modulated by the object height distribution, (3) calculating the phase modulation by analyzing the image with one of the fringe analysis techniques (such as Fourier transform

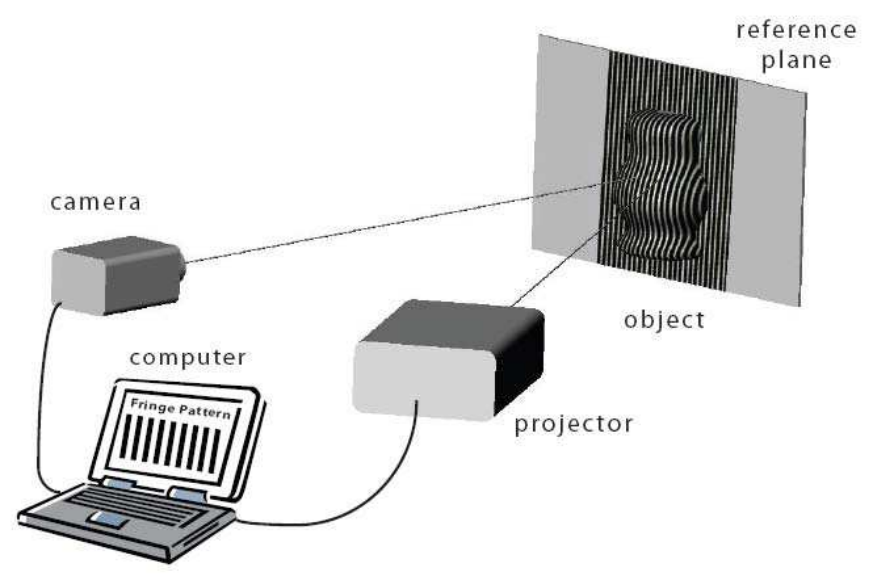

Figure 1: Fringe projection profilometry system

method, phase stepping and spatial phase detection methodsmost of them generate wrapped phase distribution) (4) using a suitable phase unwrapping algorithm to get continuous phase distribution which is proportional to the object height variations, and finally (5) calibrating the system for mapping the unwrapped phase distribution to real world 3-D co-ordinates. Fig. 2 shows the flowchart that depicts different steps involved in the measurement of height distribution of an object using the fringe projection technique and the role of each step. A pictorial representation of the same with more details is shown in Fig. 3.

During the last three decades, fringe projection techniques have developed tremendously due to the contribution of large number of researchers and the developments can be broadly categorized as follows: design or structure of the pattern used for projection [40, 41, 42, 43, 44, 45, 46, 47, 48, 49], method of generating and projecting the patterns [50, 51, 52, $53,54,55,56,57,58,59,60,61,62]$, study of errors caused by the equipment used and proposing possible corrections [63, 64, 65, 66], developing new fringe analysis methods to extract underlying phase distribution $[67,68,69,70$, $71,72,73,74,75,76,77,78,79,80,81,82,83]$, improving existing fringe analysis methods $[84,85,86,87,88,89$, 90, 91, 92, 93, 94, 95, 96, 97, 98, 99, 100], phase unwrapping algorithms [101, 102, 103, 104, 105, 106, 107, 108, 109], calibration techniques $[110,111,112,113,114,115,116,117$, $118,119,120,121,122,123]$, scale of measurement (mi- 


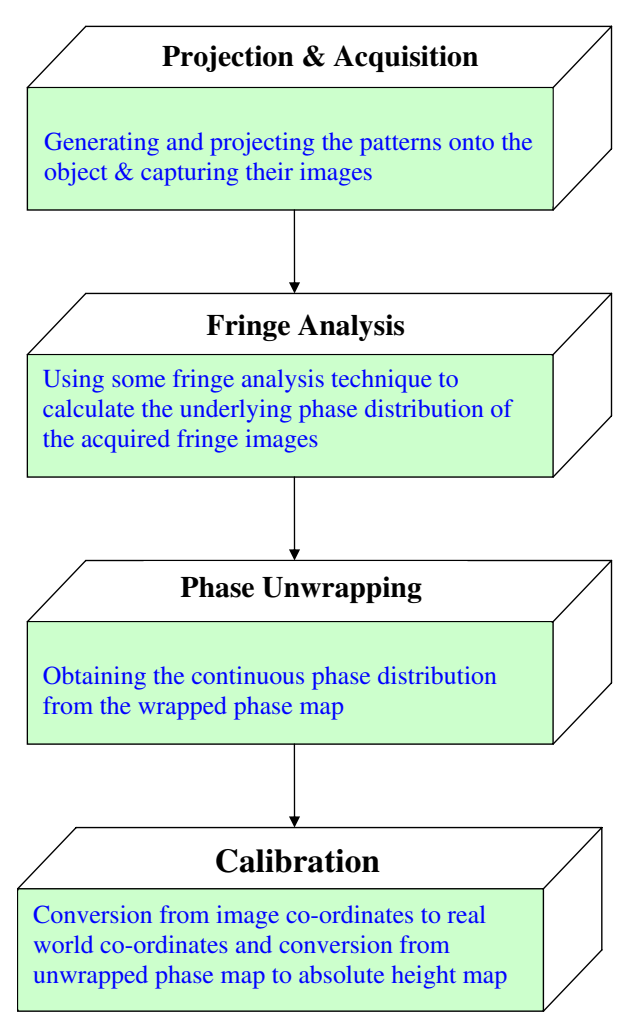

Figure 2: Flow chart

cro/medium/large objects) [124, 125, 126, 127, 128, 129, 130], state of the object (static/dynamic) [131, 132, 133, 134] and exploring the use of these techniques in diverse areas (different applications) $[1,2,3,4,5,6,7,8,9,10,11,12,13,14$, $15,16,17,18,19,20,21,22,23,24,25,26,27,28,29,30$, $31,32,33,34,35,36,37,38,39]$. Though the measurement process with the fringe projection techniques has several steps, and in each step there exists several variants, these techniques are classified mainly depending on the type of fringe analysis method that is employed. For example, based on the particular fringe analysis method used in the measurement, fringe projection techniques are classified as phase stepping profilometry (PSP), Fourier transform profilometry (FTP), wavelet transform profilometry (WTP), spatial filtering profilometry (SFP) etc.

Fringe analysis is a key task and which significantly influences the overall performance of the fringe projection profilometry system in terms of number of images required, resolution and accuracy of measurement, computational requirements etc. Over the last three decades, several fringe analysis methods have been developed. Broadly they can be categorized as spatial and temporal analysis methods. Their efficient and successful application require the presence of a sufficiently highfrequency spatial carrier for spatial methods, and acquisition of a number of images by projecting phase-shifted fringe patterns for temporal methods. Some of the fringe analysis methods introduced in the context of fringe projection profilometry are Fourier transform method and numerous extensions of it [67, 84, 85, 86, 87, 88, 89, 90], interpolated Fourier transform [68], regressive Fourier transform [135, 69], dilating Gabor transform [70], windowed Fourier transform [71, 136], multiscale windowed Fourier transform [72, 73], one-dimensional and two-dimensional Wavelet transforms [? 74, 91, 92], Stransform [75], discrete-cosine transform [76], modified Hilbert transform [77], analysis using inverse cosine function [78], neural networks [79, 137], phase locked loop [80, 93, 94, 95], regularized phase tracker $[81,138]$, spatial phase detection $[82,96$, 139], and phase-shifting methods [83, 97, 98, 99, 100]. Articles by Lei Huang et al. and C. Quan et al. in this special issue provide a comparative analysis among some of the most commonly used fringe analysis methods.

The recovered/estimated phase from the the deformed fringe pattern by using most of the aforementioned fringe analysis methods is mathematically limited to the interval $[-\pi,+\pi]$ corresponding to the principal value of arctan function. In general, the true phase may range over an interval greater than $2 \pi$ in which case the recovered phase contains artificial discontinuities. The process of determining the unknown integral multiple of $2 \pi$ to be added at each pixel of the wrapped phase map to make it continuous by removing the artificial $2 \pi$ discontinuities is referred to as phase unwrapping. Normal phase unwrapping is carried out by comparing the phase at neighboring pixels and adding or subtracting $2 \pi$ to bring the relative phase between the two pixels into the range of $-\pi$ to $+\pi$. Thus, phase unwrapping is a trivial task if the wrapped phase map is ideal. However, in real measurements, the presence of shadows, low fringe modulations, non-uniform reflectivities of the object surface, fringe discontinuities, noise etc. makes phase unwrapping difficult and path dependent. Several advanced unwrapping algorithms have been developed and some of them are: Goldstein's algorithm [140], Z $\pi \mathrm{M}$ algorithm [141], temporal phase unwrapping algorithm [101], fringe frequency analysis based algorithm [102], Fourier transform profilometry based [103], region growing phase unwrapping [104], local histogram based phase unwrapping [105], improved noise immune phase unwrapping [106], regularized phase tracking [107], flood fill [108], PEARLS (phase estimation using adaptive regularization based on local smoothing) [142], and multilevel quality guided phase unwrapping algorithm [109]. Some review articles on unwrapping include [143, 144, 145, 146]. Zappa et al. [147] performed a comparison of the performance of eight unwrapping algorithms (Goldstein's, quality guided path following, Mask cut, Flynn's, multi-grid, weighted multi-grid, preconditioned conjugate gradient and minimum Lp-norm algorithm) in the context of measuring 3D shape information using Fourier transform profilometry. In the case of dynamic/real-time 3D shape measurement, a stack of 2D wrapped phase maps obtained at different time instants needs to be unwrapped, for which a threedimensional phase unwrapping algorithm is required. For a detailed discussion on 3D phase unwrapping, refer to the review article on "Dynamic 3D shape measurement" by Xianyu Su and Qican Zhang in this special issue.

It is worth noting that, in general, the continuous phase distribution that is obtained by the analysis of deformed fringe pattern followed by the use of a phase unwrapping algorithm, contains the sum of object's shape-related phase and 


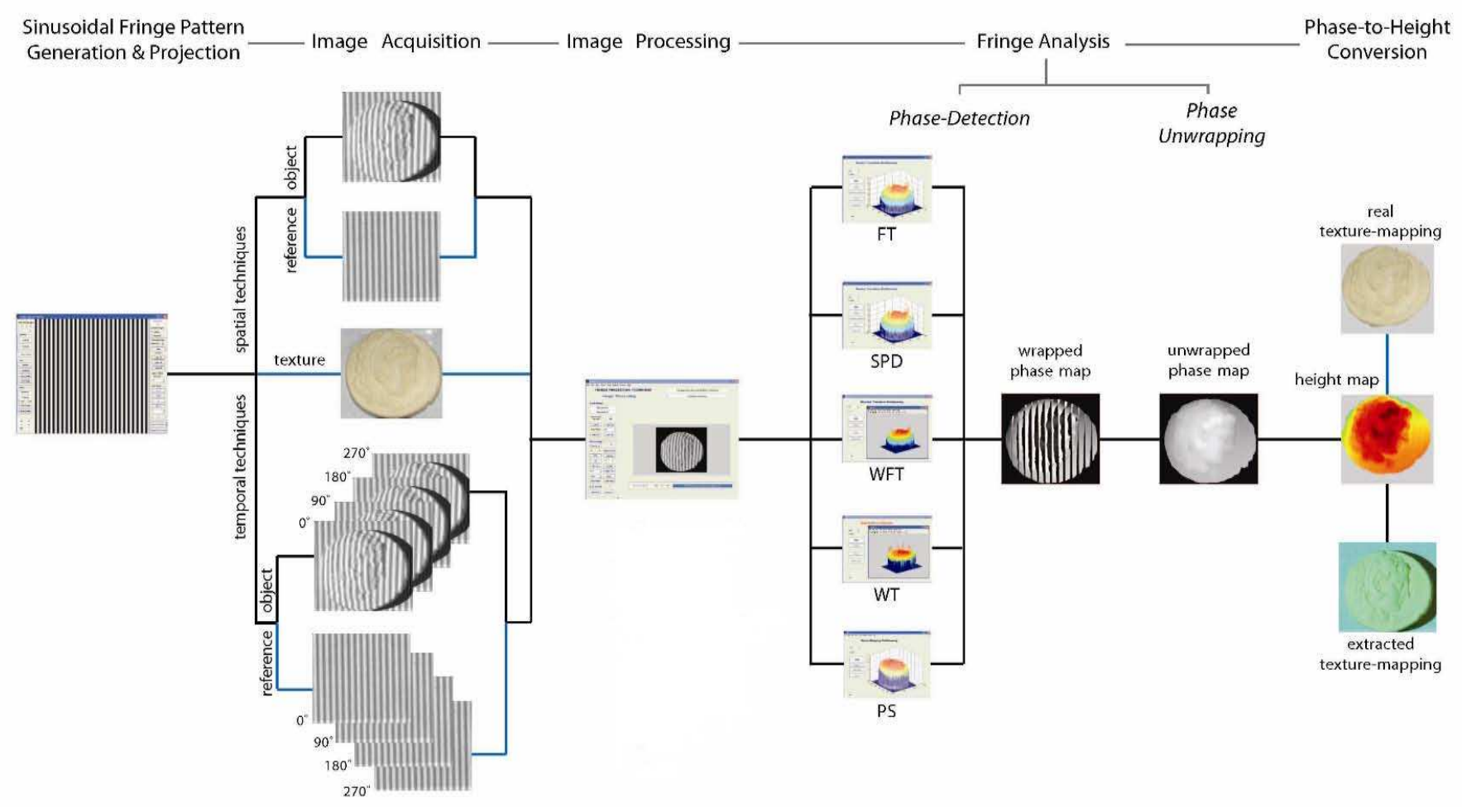

Figure 3: Work-flow in Fringe Projection Profilometry

carrier-fringe-related phase. It is thus imperative to have a method to effectively remove the carrier related-phase component, in order to accurately estimate the 3D shape of the object [148]. For a detailed comparison of the linear carrier removal techniques (spectrum-shift approach, average-slope approach, plane-fitting approach) and nonlinear carrier removal techniques (reference-subtraction approach, phase-mapping approach, series-expansion approach) refer to [149].

The last important step in the process of measuring the 3D height distribution using fringe projection technique is system calibration. It facilitates the conversion of image coordinates (pixels) to the real-world coordinates and the mapping of unwrapped phase distribution to the absolute height distribution. The former task is often accomplished by adopting standard camera calibration techniques of the computer vision field $[150,151,152]$. It normally involves, determining the intrinsic parameters of the camera and extrinsic parameters that describe the transformation relationship between the 3D world coordinates and 2D image coordinates. For the later task, ideally in an off-axis optical setup, simple triangulation principle establishes the relation between the unwrapped phase distribution and the geometric coordinates of the $3 \mathrm{D}$ object. The relation that governs this conversion is given by [67]:

$$
h(x, y)=\frac{l_{0} \Delta \phi(x, y)}{\Delta \phi(x, y)+2 \pi f_{0} d}
$$

The above equation can be rewritten as:

$$
h(x, y)=\frac{\Delta \phi(x, y)}{C_{1}(x, y)+C_{2}(x, y) \Delta \phi(x, y)}
$$

where $d$ and $l_{0}$ are the parameters of the optical setup. $d$ represents the distance between the entrance pupil of the camera (C) and the exit pupil of the projector $(\mathrm{P})$. $\mathrm{C}$ and $\mathrm{P}$ are assumed to be in a parallel plane at a distance $l_{0}$ from the reference plane. $f_{0}$ represents the spatial frequency of the projected fringe pattern. $\Delta \phi(x, y)$ is the object shape-related phase component obtained after removing the carrier-related phase component as mentioned in the preceding paragraph. $C_{1}(x, y)$ and $C_{2}(x, y)$ are optical setup related coefficients that need to be estimated using calibration techniques. In linear calibration methods, based on the assumption that $l_{0}$ is much larger than $h$, Eq. (1) is approximated with a linear relation:

$$
h(x, y)=\frac{l_{0} \Delta \phi(x, y)}{2 \pi f_{0} d}=K(x, y) \Delta \phi(x, y)
$$

where $K(x, y)$ is the calibration coefficient. It is a function of the spatial coordinates $(x, y)$ and is decided by the parameters of the optical setup. However, in practical conditions, it is very difficult to precisely measure system parameters such as $l_{0}, d$ and even more difficult is the measurement of $f_{0}$ as it does not remain constant over the entire image plane due to the nontelecentric projection (divergence of the projector rays). Various calibration techniques have emerged as solutions to this problem. Without manually measuring these parameters, by capturing few additional images and analyzing them, nonlinear calibration techniques provide estimates for $C_{1}(x, y), C_{2}(x, y)$, and linear calibration techniques provide estimate for $K(x, y)$, thereby enabling one to calculate $h(x, y)$ using either Eq. (2) or Eq. (3) respectively. For a comparison of the performance of 


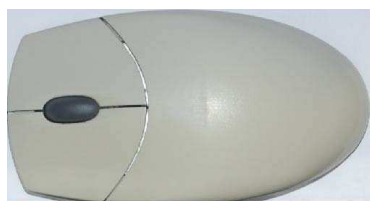

(a)

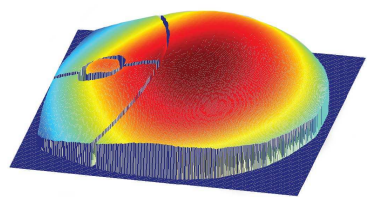

(c)

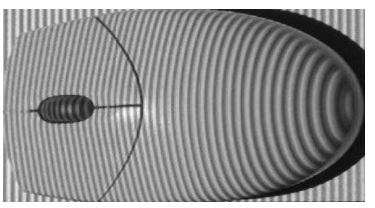

(b)

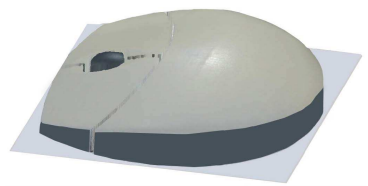

(d)

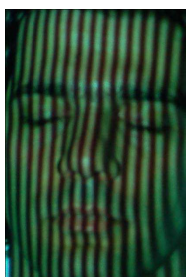

(a)

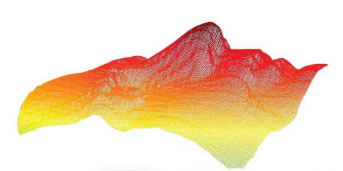

(c)

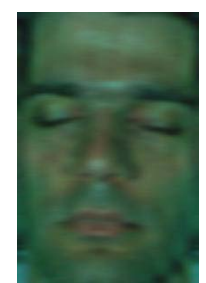

(b)

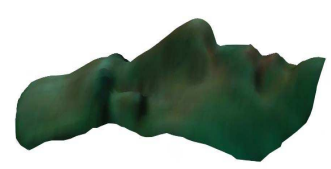

(d)
Figure 4: (a) Image of the object without projecting the fringes (captured to have the real texture), (b) Image of the object recorded by projecting the fringe pattern, (c) mesh plot of the recovered 3D shape distribution of the object obtained by analyzing (b), and (d) Texture mapping onto the estimated 3D shape distribution to give a realistic view of the $3 \mathrm{D}$ object

the linear and nonlinear calibration techniques refer to [153]. Several calibration techniques have been developed over the years $[110,111,112,113,114,115,116,117,118]$ and recently there has been an increasing interest in the development of flexible/generalized calibration techniques [119, 120, 121, $122,123]$ that are capable of automatically determining the geometric parameters of the experimental setup even when the camera, projector and object are placed arbitrarily (i.e., by relaxing the constraint of following the conventional in-line and off-axis optical geometries).

Texture mapping is an optional posterior operation to the 3D shape estimation that is often performed. It has an important utility in applications such as face recognition, graphics and gaming, as it produces a realistic view of the reconstructed 3D object. In addition to providing an aesthetic view for the reconstructed 3D shape, texture mapping also serves to generate 'unseen' 2D views of the object [35]. This is of crucial importance in face recognition applications as many recognition approaches rely on multiple views of the face as inputs [154]. If the application allows the recording of an additional image of the object without projecting the fringe pattern (see Fig. 4a), then this recorded real-texture information can be mapped onto the estimated $3 \mathrm{D}$ shape distribution as shown in Fig. 4d. In the case of dynamic measurements, it may not be feasible to capture (in each state) separate images of the object with and without the projection of fringes. In such cases, extracting the texture map from the fringe-projected-image itself is an attractive solution. It can be accomplished by performing simple lowpass filtering operation along each color channel and merging them together. Fig. 5a shows the image of the face with the fringe pattern projected. Fig. 5b shows the texture map extracted from Fig. 5a. The plots of the estimated 3D shape distribution before and after texture mapping are shown in Fig. 5c and Fig. 5d respectively.

Most of the present day automated 3D measurement sys-
Figure 5: (a) Image of the face recorded by projecting the fringe pattern, (b) Texture extracted from Fig. 5a by low-pass filtering along each color channel, (c) Mesh plot of the recovered 3D shape distribution obtained by analyzing Fig. 5a, and (d) Estimated 3D shape distribution with texture mapping

tems employ the commercially available digital micromirror device (DMD) and liquid crystal display (LCD) projectors for projecting the computer generated fringe patterns onto the object. However, for various reasons, several other methods have also been in use for the generation and projection of sinusoidal fringes. For example, fringes generated by conventional interferometric techniques such as Michelson interferometer are often used for projecting high-frequency fringe patterns [50, 51]. Fiber optic interferometer system with the laser diode input enables one to have greater flexibility and compactness of the measurement system [52]. Grating projection systems [53, 54], spatial light modulators [55], diffractive optical elements [56], superluminiscent diode in conjunction with an acousto-optic tunable filter [57], programmable LCDs [45], multi-core optical fibers $[58,59]$ etc. are among the other methods that are in practice used to generate and project structured patterns onto the objects. It is interesting to note that most of these methods can also be employed to generate phase-shifted fringe patterns $[60,61,62]$.

Nevertheless, LCD and DMD projectors provide great flexibility in projecting any kind of computer generated patterns (not necessarily sinusoidal fringe pattern). This feature has allowed researchers to come up with design of various kinds of structured patterns and associated processing algorithms for measuring the 3D shapes of objects [40]. Some of the related works include the use of hexagonal grating [41], saw-tooth fringe pattern [42, 43], triangular pattern [44], locally adapted projection $[45,46]$, the development of inverse function analysis method that permits projection of any kind of fringe pattern [47], gray-code light projection [48], optimal intensitymodulation projection [49] etc.

The use of multiple projectors in fringe projection profilometry has also been reported to solve some of the baffling problems like the accurate 3D estimation in presence of local shadows, invalid regions and surface isolations $[155,156,157,158]$. In 
addition, it has opened up new avenues for in-situ quality testing of industrial components/products by supporting techniques like inverse projected-fringes [159].

While there exists quite a large number of reports on the use of fringe projection techniques for measuring 3D shapes of medium-size objects, a moderate number of reports are available on its use in micro-scale measurements [12, 13, 23, $124,125,126,127]$ and very little work has been reported in large-scale measurements (spanning few square meters of area) $[128,129,130]$. Lack of proper methods for calibration and for removing the carrier-phase component from the measured phase are among the potential reasons that are responsible for the limited use of the technique in micro-scale and large-scale measurements.

The presence of non-sinusoidal waveforms in the recorded fringe patterns is known to cause significant phase measurement errors (when phase-shifting analysis method is employed) and thereby resulting in non-negligible errors in the measurement of 3D shapes of objects. It is largely due to the nonlinearity of the projector's gamma [160] and the nonlinearity of the CCD camera that the recorded fringe patterns are observed to have harmonics (non-sinusoidal waveforms). Recently, due attention has been paid by different researchers [161, 162] to compensate for the errors caused by the nonlinear gamma of the projector such as by proposing the use of pre-calibrated look up tables [163]. Not only the results of phase shifting methods but also that of spatial analysis methods have been recently noticed to be influenced by the presence of harmonics [164] (see also the article by Lei Huang et al. in this special issue). Thus one has to take due consideration of the presence of harmonics while measuring 3D shapes of objects with the fringe projection techniques.

Fringe projection techniques that employ phase-stepping method for the fringe analysis are known to provide highresolution $3 \mathrm{D}$ reconstruction with minimal computational investment. However, it requires multiple images (at least 3) that are recorded by projecting a sequence of phase-shifted fringe patterns. This requirement restricts their applicability to static objects. Spatial fringe analysis methods on the other hand allow the technique to be applicable for real-time 3D measurements but at the cost of resolution. In view of this, efforts have been made to boost the measurement speed of phase-shifting profilometry; as a result multichannel approach has emerged as a potential solution [165]. Huang et al. [166] proposed a method in which color encoded fringes are projected which enables to obtain 3D surface contour of the object from a single snapshot. In this method, a color fringe pattern with RGB (red, green, and blue) components comprising of the three phase-shifted fringe patterns is generated in a computer and projected using a digital projector. The deformed color-fringe image is captured by a color CCD camera and then separated into its RGB components, thereby effectively creating three phase-shifted deformed fringe images. These three images are then used to retrieve the 3D height distribution of the object using three-bucket phaseshifting algorithm. As each color (channel) is carrying different phase-shifted patterns, it is referred to as multichannel approach. Since the color information is used to separate three phase-shifted fringe patterns, coupling effect between channels, also referred to as color channel crosstalk, affects the measurement results [167]. Therefore, color isolation process is the inevitable precursor to the phase-shifting algorithm to effectively reconstruct the 3D shape in the multichannel approach. To tackle this problem, different solutions have been proposed including: linear mixing model with the associated calibration method [168], blind signal separation algorithm based calibration method [169] and blind color isolation method that is capable of determining color demixing matrix without requiring any additional images for color calibration [170].

Accurate and unambiguous 3D shape measurement of $o b$ jects having surface discontinuities or spatially isolated surfaces is one of the most challenging problems in fringe projection profilometry. It is essentially because of projecting a sinusoidal fringe pattern (for that matter any periodic pattern) that the obtained wrapped phase map with the fringe analysis contains true phase discontinuities as well (i.e., discontinuities in the wrapped phase map that are originating from the object surface discontinuities) and the correct unwrapping of it is impossible from a single measurement. To overcome this problem, several phase unwrapping strategies have been developed. Multi-sensitivity based unwrapping [171, 172], temporal phase unwrapping [101], reduced temporal phase unwrapping [173] and spatio-temporal phase unwrapping [174] among them are the most popular ones. However, to perform phase unwrapping, all these methods mandatorily require multiple phase maps generated by varying the spatial frequency of projected fringe pattern either linearly or exponentially. Further, the degree of reliability varies from method to method [175, 176]. It is desirable to be able to estimate the $3 \mathrm{D}$ shape information from a single image, as the measurements can be corrupted by the movements of the object during the projection/capture process. To meet this demand, another class of structured light techniques based on color-coded pattern projection is developed, which provides from a single image, unambiguous reconstruction of 3D shape but at reduced spatial and depth resolutions [177, 178]. The third class of techniques has been introduced, in which the designed pattern encodes features of the above two classes and thereby enables in retrieving highresolution unambiguous $3 \mathrm{D}$ shape information from a single image. Some of the methods in this class use HSI (hue, saturation, and intensity) color model and incorporate coding strategy in hue-channel and sinusoidal variations in intensitychannel [179, 180, 181, 182, 183]. Few other techniques which effectively combine multiple-frequency patterns into a single composite pattern for projection, thereby allowing for real-time measurements can be found in [184, 185, 186, 187, 188].

Last but not the least, this special issue with its fifteen papers, including the four review articles, provides a good exposure to the state of the art by touching upon different facets of the fringe projection techniques. We hope this issue will serve as a valuable source of reference in the field to both the budding researcher and the professional alike. 


\section{Acknowledgments}

S. S. Gorthi would like to express his deep gratitude to Dr. L. Kameswara Rao, Department of Instrumentation, Indian Institute of Science, Bangalore, India, for providing valuable insights in the field. We would like to thank Mr. Gianfranco Bianco for his assistance in the preparation of illustrations.

\section{References}

[1] L. Chen, C. Huang, Miniaturized 3D surface profilometer using digital fringe projection, Meas. Sci. Techn. 16 (5) (2005) 1061-1068.

[2] K. Genovese, C. Pappalettere, Whole 3D shape reconstruction of vascular segments under pressure via fringe projection techniques, Opt. Laser Eng. 44 (12) (2006) 1311-1323.

[3] F. Lilley, M. J. Lalor, D. R. Burton, Robust fringe analysis system for human body shape measurement, Opt. Eng. 39 (1) (2000) 187-195.

[4] C. J. Moore, D. R. Burton, O. Skydan, P. J. Sharrock, M. Lalor, 3D body surface measurement and display in radiotherapy part I: Technology of structured light surface sensing, Proc. Int. Confe. Medical Information Visualisation - BioMedical Visualisation (1691277) (2006) 97-102.

[5] A. Hanafi, T. Gharbi, J. Cornu, In vivo measurement of lower back deformations with Fourier-transform profilometry, Appl. Opt. 44 (12) (2005) 2266-2273.

[6] F. Berryman, P. Pynsent, J. Fairbank, S. Disney, A new system for measuring three-dimensional back shape in scoliosis, European Spine Journal 17 (5) (2008) 663-672.

[7] T. Hain, R. Eckhardt, K. Kunzi-Rapp, B. Schmitz, Indications for optical shape measurements in orthopaedics and dermatology, Medical Laser Application 17 (1) (2002) 55-58.

[8] Y. Ferraq, D. Black, J. M. Lagarde, A. M. Schmitt, S. Dahan, J. L. Grolleau, S. Mordon, Use of a 3-D imaging technique for non-invasive monitoring of the depth of experimentally induced wounds, Skin Research and Technology 13 (4) (2007) 399-405.

[9] S. Jaspers, H. Hopermann, G. Sauermann, U. Hoppe, R. Lunderstädt, J. Ennen, Rapid in vivo measurement of the topography of human skin by active image triangulation using a digital micromirror device, Skin Research and Technology 5 (3) (1999) 195-207.

[10] J. M. Lagarde, C. Rouvrais, D. Black, S. Diridollou, Y. Gall, Skin topography measurement by interference fringe projection: A technical validation, Skin Research and Technology 7 (2) (2001) 112-121.

[11] http://www.diip-online.net/DOCUMENTS/EOTECH-Surface-TextureShape3D-2007-en.pdf

[12] C. Quan, C. J. Tay, X. Y. He, X. Kang, H. M. Shang, Microscopic surface contouring by fringe projection method, Opt. Laser Techn. 34 (7) (2002) $547-552$.

[13] X. He, W. Sun, X. Zheng, M. Nie, Static and dynamic deformation measurements of micro beams by the technique of digital image correlation, Key Engineering Materials 326-328 (2006) 211-214.

[14] S. T. Yilmaz, U. D. Ozugurel, K. Bulut, M. N. Inci, Vibration amplitude analysis with a single frame using a structured light pattern of a four-core optical fibre, Opt. Commun. 249 (4-6) (2005) 515-522.

[15] Q. Zhang, X. Su, High-speed optical measurement for the drumhead vibration, Opt. Express 13 (8) (2005) 3110-3116.

[16] M. De Angelis, S. De Nicola, P. Ferraro, A. Finizio, G. Pierattini, Liquid refractometer based on interferometric fringe projection, Opt. Commun. 175 (4) (2000) 315-321.

[17] Q. Zhang, X. Su, An optical measurement of vortex shape at a free surface, Opt. Laser Technol. 34 (2) (2002) 107-113.

[18] P. J. Cobelli, A. Maurel, V. Pagneux, P. Petitjeans, Global measurement of water waves by Fourier transform profilometry, Experiments in Fluids 46 (6) (2009) 1037-1047.

[19] R. V. Roger Ernst, Albert Weckenmann, Local wall thickness measurement of formed sheet metal using fringe projection technique, in: Proceedings, XVII IMEKO World Congress, 1802-1805, 2003.

[20] P. S. Huang, F. Jin, F. Chiang, Quantitative evaluation of corrosion by a digital fringe projection technique, Opt. Laser Eng. 31 (5) (1999) 371380 .
[21] P. Jang, et al, Quantitative imaging characterization of aluminum pit corrosion in Oak Ridge research reactor pool, Proc. SPIE 6377 (63770S) (2006).

[22] G. S. Spagnolo, D. Ambrosini, Diffractive optical element based sensor for roughness measurement, Sensors and Actuators A: Physical 100 (23) (2002) 180-186.

[23] L. Chen, Y. Chang, High accuracy confocal full-field 3-D surface profilometry for micro lenses using a digital fringe projection strategy, Key Engineering Materials 364-366 (2008) 113-116.

[24] J. Burke, T. Bothe, W. Osten, C. Hess, Reverse engineering by fringe projection, Proc. SPIE 4778 (2002) 312-324.

[25] C. Lin, H. He, H. Guo, M. Chen, X. Shi, T. Yu, Fringe projection measurement system in reverse engineering, Journal of Shanghai University 9 (2) (2005) 153-158.

[26] J. Hecht, K. Lamprecht, M. Merklein, K. Galanulis, J. Steinbeck, Triangulation based digitizing of tooling and sheet metal part surfaces Measuring technique, analysis of deviation to CAD and remarks on use of 3D-coordinate fields for the finite element analysis, Key Engineering Materials 344 (2007) 847-853.

[27] H. Yen, D. Tsai, J. Yang, Full-field 3-D measurement of solder pastes using LCD-based phase shifting techniques, IEEE Trans. Electronics Packaging Manufacturing 29 (1) (2006) 50-57.

[28] T. Hui, G. K. Pang, Solder paste inspection using region-based defect detection, Int. Journal of Advanced Manufacturing Technology 42 (7-8) (2009) 725-734.

[29] D. Hong, H. Lee, M. Y. Kim, H. Cho, J. Moon, Sensor fusion of phase measuring profilometry and stereo vision for three-dimensional inspection of electronic components assembled on printed circuit boards, Appl. Opt. 48 (21) (2009) 4158-4169.

[30] D. Ambrosini, D. Paoletti, Heat transfer measurement by a diffractive optical element fringe projection, Opt. Eng. 46 (9) (2007) 093606.

[31] S. Tan, D. Song, L. Zeng, Tracking fringe method for measuring the shape and position of a swimming fish, Opt. Commun. 173 (1-6) (2000) 123-128.

[32] F. Yuan, D. Song, L. Zeng, Measuring 3D profile and position of a moving object in large measurement range by using tracking fringe pattern, Opt. Commun. 196 (1-6) (2001) 85-91.

[33] G. Wu, L. Zeng, L. Ji, Measuring the Wing Kinematics of a Moth (Helicoverpa Armigera) by a Two-Dimensional Fringe Projection Method, Journal of Bionic Engineering 5 (2008) 138-142.

[34] P. Cheng, J. Hu, G. Zhang, L. Hou, B. Xu, X. Wu, Deformation measurements of dragonfly's wings in free flight by using Windowed Fourier Transform, Opt. Laser Eng. 46 (2) (2008) 157-161.

[35] J. Yagnik, S. S. Gorthi, K. R. Ramakrishnan, L. K. Rao, 3D shape extraction of human face in presence of facial hair: A profilometric approach, Proc. IEEE Region 10 Annual International Conference (4085277) (2007).

[36] G. Zhou, Z. Li, C. Wang, Y. Shi, A Novel Method for Human Expression Rapid Reconstruction, Tsinghua Science and Technology 14 (2009) 6265.

[37] G. Guidi, M. Pieraccini, S. Ciofi, V. Damato, J. Beraldin, C. Atzeni, Tridimensional digitizing of Donatello's Maddalena, in: IEEE Int. Conf. Image Processing, vol. 1, 578-581, 2001.

[38] G. S. Spagnolo, D. Ambrosini, D. Paoletti, Low-cost optoelectronic system for three-dimensional artwork texture measurement, IEEE Trans. Image Processing 13 (3) (2004) 390-396.

[39] G. Sansoni, F. Docchio, 3-D optical measurements in the field of cultural heritage: The case of the Vittoria Alata of Brescia, IEEE Trans. Instrumentation and Measurement 54 (1) (2005) 359-368.

[40] J. Salvi, J. Pages, J. Batlle, Pattern codification strategies in structured light systems, Pattern Recognition 37 (4) (2004) 827-849.

[41] K. Iwata, F. Kusunoki, K. Moriwaki, H. Fukuda, T. Tomii, Threedimensional profiling using the fourier transform method with a hexagonal grating projection, Appl. Opt. 47 (12) (2008) 2103-2108.

[42] Q. Fang, S. Zheng, Linearly coded profilometry, Appl. Opt. 36 (11) (1997) 2401-2407.

[43] L. Chen, C. Quan, C. J. Tay, Y. Fu, Shape measurement using one frame projected sawtooth fringe pattern, Opt. Commun. 246 (4-6) (2005) 275284.

[44] P. Jia, J. Kofman, C. English, Error compensation in two-step triangularpattern phase-shifting profilometry, Opt. Laser Eng. 46 (4) (2008) 311- 
320

[45] M. K. Kalms, W. P. Jueptner, W. Osten, Automatic adaption of projected fringe patterns using a programmable LCD-projector, Proc. SPIE 3100 (1997) $156-165$

[46] B. Denkena, W. Acker, Three-dimensional optical measurement with locally adapted projection, Advanced Materials Research 22 (2007) 8390.

[47] Y. Hu, J. Xi, J. F. Chicharo, W. Cheng, Z. Yang, Inverse Function Analysis Method for Fringe Pattern Profilometry, IEEE Trans. Instrumentation and Measurement Article in Press.

[48] G. Sansoni, S. Corini, S. Lazzari, R. Rodella, F. Docchio, Threedimensional imaging based on Gray-code light projection: Characterization of the measuring algorithm and development of a measuring system for industrial applications, Appl. Opt. 36 (19) (1997) 4463-4472.

[49] C. Lu, L. Xiang, Optimal intensity-modulation projection technique for three-dimensional shape measurement, Appl. Opt. 42 (23) (2003) 46494657.

[50] E. B. Li, X. Peng, J. Xi, J. F. Chicharo, J. Q. Yao, D. W. Zhang, Multifrequency and multiple phase-shift sinusoidal fringe projection for 3D profilometry, Opt. Express 13 (5) (2005) 1561-1569.

[51] J. I. Harizanova, E. V. Stoykova, V. C. Sainov, Phase retrieval techniques in coordinates measurement, in: AIP Conference Proceedings, vol. 899, 321-322, 2007.

[52] T. L. Pennington, H. Xiao, R. May, A. Wang, Miniaturized 3-D surface profilometer using a fiber optic coupler, Opt. Laser Technol. 33 (5) (2001) 313-320.

[53] W. Su, K. Reichard, S. Yin, F. T. S. Yu, Fabrication of digital sinusoidal gratings and precisely controlled diffusive flats and their application to highly accurate projected fringe profilometry, Opt. Eng. 42 (6) (2003) $1730-1740$

[54] J. Zhang, C. Zhou, X. Wang, Three-dimensional profilometry using a Dammann grating, Appl. Opt. 48 (19) (2009) 3709-3715.

[55] C. R. Coggrave, J. M. Huntley, High-speed surface profilometer based on a spatial light modulator and pipeline image processor, Opt. Eng. 38 (9) (1999) 1573-1581.

[56] G. S. Spagnolo, D. Ambrosini, Diffractive optical element-based profilometer for surface inspection, Opt. Eng. 40 (1) (2001) 44-52.

[57] T. Anna, S. K. Dubey, C. Shakher, A. Roy, D. S. Mehta, Sinusoidal fringe projection system based on compact and non-mechanical scanning low-coherence Michelson interferometer for three-dimensional shape measurement, Opt. Commun. 282 (7) (2009) 1237-1242.

[58] K. Bulut, M. N. Inci, Three-dimensional optical profilometry using a four-core optical fibre, Opt. Laser Technol. 37 (6) (2005) 463-469.

[59] L. Yuan, J. Yang, C. Guan, Q. Dai, F. Tian, Three-core fiber-based shapesensing application, Opt. Lett. 33 (6) (2008) 578-580.

[60] H. Fan, H. Zhao, Y. Tan, Automated three-dimensional surface profilometry using dual-frequency optic fiber phase-shifting method, Opt. Eng. 36 (11) (1997) 3167-3171.

[61] F. Wu, H. Zhang, M. J. Lalor, D. R. Burton, Novel design for fiber optic interferometric fringe projection phase-shifting 3-D profilometry, Opt. Commun. 187 (4-6) (2001) 347-357.

[62] M. Yokota, A. Asaka, T. Yoshino, Stabilization improvements of laserdiode closed-loop heterodyne phase-shifting interferometer for surface profile measurement, Appl. Opt. 42 (10) (2003) 1805-1807.

[63] P. S. Huang, Q. Hu, F. Chiang, Error compensation for a threedimensional shape measurement system, Opt. Express 42 (2) (2003) 482-486.

[64] Z. Zhang, D. Zhang, X. Peng, Performance analysis of a 3D full-field sensor based on fringe projection, Opt. Laser Eng. 42 (3) (2004) 341353.

[65] P. Brakhage, G. Notni, R. Kowarschik, Image aberrations in optical three-dimensional measurement systems with fringe projection, Appl. Opt. 43 (16) (2004) 3217-3223.

[66] H. Guo, H. He, M. Chen, Gamma correction for digital fringe projection profilometry, Appl. Opt. 43 (14) (2004) 2906-2914.

[67] M. Takeda, K. Mutoh, Fourier transform profilometry for the automatic measurement of 3-D object shapes., Appl. Opt. 22 (24) (1983) 39773982.

[68] S. Vanlanduit, J. Vanherzeele, P. Guillaume, B. Cauberghe, P. Verboven, Fourier fringe processing by use of an interpolated Fourier-transform technique, Appl. Opt. 43 (27) (2004) 5206-5213.
[69] J. Vanherzeele, P. Guillaume, S. Vanlanduit, Fourier fringe processing using a regressive Fourier-transform technique, Opt. Laser Eng. 43 (6) (2005) 645-658.

[70] J. Zhong, J. Weng, Dilating Gabor transform for the fringe analysis of 3-D shape measurement, Opt. Eng. 43 (4) (2004) 895-899.

[71] Q. Kemao, Windowed Fourier transform for fringe pattern analysis, Appl. Opt. 43 (13) (2004) 2695-2702.

[72] S. Zheng, W. Chen, X. Su, Adaptive windowed Fourier transform in 3-D shape measurement, Opt. Eng. 45 (6) (2006) 063601.

[73] J. Zhong, H. Zeng, Multiscale windowed Fourier transform for phase extraction of fringe patterns, Appl. Opt. 46 (14) (2007) 2670-2675.

[74] A. Dursun, S. Ozder, F. N. Ecevit, Continuous wavelet transform analysis of projected fringe patterns, Meas. Sci. Techn. 15 (9) (2004) 1768 1772.

[75] S. Ozder, O. Kocahan, E. Coskun, H. Goktas, Optical phase distribution evaluation by using an S-transform, Opt. Lett. 32 (6) (2007) 591-593.

[76] Y. Hu, J. Xi, J. Chicharo, E. Li, Z. Yang, Discrete cosine transformbased shift estimation for fringe pattern profilometry using a generalized analysis model, Appl. Opt. 45 (25) (2006) 6560-6567.

[77] M. A. Sutton, W. Zhao, S. R. McNeill, H. W. Schreier, Y. J. Chao, Development and assessment of a single-image fringe projection method for dynamic applications, Experimental Mechanics 41 (3) (2001) 205-217.

[78] K. Okada, E. Yokoyama, H. Miike, Interference fringe pattern analysis using inverse cosine function, Electronics and Communications in Japan, Part II: Electronics 90 (1) (2007) 61-73.

[79] Y. Tangy, W. Chen, X. Su, L. Xiang, Neural network applied to reconstruction of complex objects based on fringe projection, Opt. Commun. 278 (2) (2007) 274-278

[80] R. Rodríguez-Vera, M. Servín, Phase locked loop profilometry, Opt. Laser Techn. 26 (6) (1994) 393-398.

[81] J. Villa, M. Servin, Robust profilometer for the measurement of 3-D object shapes based on a regularized phase tracker, Opt. Laser Eng. 31 (4) (1999) 279-288.

[82] S. Toyooka, Y. Iwaasa, Automatic profilometry of 3-D diffuse objects by spatial phase detection, Appl. Opt. 25 (10) (1986) 1630-1633.

[83] V. Srinivasan, H. C. Liu, M. Halioua, Automated phase-measuring profilometry of 3-D diffuse objects., Appl. Opt. 23 (18) (1984) 3105-3108.

[84] J.-F. Lin, X.-Y. Su, Two-dimensional Fourier transform profilometry for the automatic measurement of three-dimensional object shapes, Opt. Eng. 34 (11) (1995) 3297-3302.

[85] X. Su, W. Chen, Fourier transform profilometry: A review, Opt. Laser Eng. 35 (5) (2001) 263-284.

[86] F. Berryman, P. Pynsent, J. Cubillo, The effect of windowing in Fourier transform profilometry applied to noisy images, Opt. Laser Eng. 41 (6) (2004) 815-825.

[87] M. A. Gdeisat, D. R. Burton, M. J. Lalor, Eliminating the zero spectrum in Fourier transform profilometry using a two-dimensional continuous wavelet transform, Opt. Commun. 266 (2) (2006) 482-489.

[88] P. J. Tavares, M. A. Vaz, Orthogonal projection technique for resolution enhancement of the Fourier transform fringe analysis method, Opt. Commun. 266 (2) (2006) 465-468.

[89] S. Li, X. Su, W. Chen, L. Xiang, Eliminating the zero spectrum in Fourier transform profilometry using Empirical mode decomposition, J. Opt. Soc. Am. A 26 (5) (2009) 1195-1201.

[90] M. Dai, Y. Wang, Fringe extrapolation technique based on Fourier transform for interferogram analysis with the definition, Opt. Lett. 34 (7) (2009) 956-958.

[91] J. Zhong, J. Weng, Spatial carrier-fringe pattern analysis by means of wavelet transform: Wavelet transform profilometry, Appl. Opt. 43 (26) (2004) 4993-4998.

[92] M. A. Gdeisat, D. R. Burton, M. J. Lalor, Spatial carrier fringe pattern demodulation by use of a two-dimensional continuous wavelet transform, Appl. Opt. 45 (34) (2006) 8722-8732.

[93] J. Kozlowski, G. Serra, New modified phase locked loop method for fringe pattern demodulation, Opt. Eng. 36 (7) (1997) 2025-2030.

[94] D. Ganotra, J. Joseph, K. Singh, Second- and first-order phase-locked loops in fringe profilometry and application of neural networks for phase-to-depth conversion, Opt. Commun. 217 (1-6) (2003) 85-96.

[95] M. A. Gdeisat, D. R. Burton, M. J. Lalor, Fringe-pattern demodulation using an iterative linear digital phase locked loop algorithm, Opt. Laser Eng. 43 (7) (2005) 31-39. 
[96] M. R. Sajan, C. J. Tay, H. M. Shang, A. Asundi, Improved spatial phase detection for profilometry using a TDI imager, Opt. Commun. 150 (1-6) (1998) 66-70.

[97] M. Chang, D. Wan, On-line automated phase-measuring profilometry, Opt. Laser Eng. 15 (2) (1991) 127-139.

[98] X. Su, W. Zhou, G. von Bally, D. Vukicevic, Automated phasemeasuring profilometry using defocused projection of a Ronchi grating, Opt. Commun. 94 (6) (1992) 561-573.

[99] X. Su, G. von Bally, D. Vukicevic, Phase-stepping grating profilometry: utilization of intensity modulation analysis in complex objects evaluation, Opt. Commun. 98 (1-3) (1993) 141-150.

[100] X. F. Meng, X. Peng, L. Z. Cai, A. M. Li, J. P. Guo, Y. R. Wang, Wavefront reconstruction and three-dimensional shape measurement by two-step dc-term-suppressed phase-shifted intensities, Opt. Lett. 34 (8) (2009) 1210-1212.

[101] H. O. Saldner, J. M. Huntley, Temporal phase unwrapping: Application to surface profiling of discontinuous objects, Appl. Opt. 36 (13) (1997) 2770-2775.

[102] S. Su, X. Lian, Phase unwrapping algorithm based on fringe frequency analysis in Fourier-transform profilometry, Opt. Eng. 40 (4) (2001) 637643.

[103] C. Yu, Q. Peng, A correlation-based phase unwrapping method for Fourier-transform profilometry, Opt. Laser Eng. 45 (6) (2007) 730-736.

[104] A. Baldi, Phase unwrapping by region growing, Appl. Opt. 42 (14) (2003) 2498-2505.

[105] J. Meneses, T. Gharbi, P. Humbert, Phase-unwrapping algorithm for images with high noise content based on a local histogram, Appl. Opt. 44 (7) (2005) 1207-1215.

[106] R. Cusack, J. M. Huntley, H. T. Goldrein, Improved noise-immune phase-unwrapping algorithm, Appl. Opt. 34 (5) (1995) 781-789.

[107] M. Servin, F. J. Cuevas, D. Malacara, J. L. Marroquin, R. RodriguezVera, Phase unwrapping through demodulation by use of the regularized phase-tracking technique, Appl. Opt. 38 (10) (1999) 1934-1941.

[108] A. Asundi, Z. Wensen, Fast phase-unwrapping algorithm based on a gray-scale mask and flood fill, Appl. Opt. 37 (23) (1998) 5416-5420.

[109] S. Zhang, X. Li, S. Yau, Multilevel quality-guided phase unwrapping algorithm for real-time three-dimensional shape reconstruction, Appl. Opt. 46 (1) (2007) 50-57.

[110] W. Schreiber, G. Notni, Theory and arrangements of self-calibrating whole-body three-dimensional measurement systems using fringe projection technique, Opt. Eng. 39 (1) (2000) 159-169.

[111] Y. Y. Hung, L. Lin, H. M. Shang, B. G. Park, Practical three-dimensional computer vision techniques for full-field surface measurement, Opt. Eng. 39 (1) (2000) 143-149.

[112] H. Liu, W. Su, K. Reichard, S. Yin, Calibration-based phase-shifting projected fringe profilometry for accurate absolute 3D surface profile measurement, Opt. Commun. 216 (1-3) (2003) 65-80.

[113] H. Guo, H. He, Y. Yu, M. Chen, Least-squares calibration method for fringe projection profilometry, Opt. Eng. 44 (3) (2005) 1-9.

[114] X. Zhang, Y. Lin, M. Zhao, X. Niu, Y. Huang, Calibration of a fringe projection profilometry system using virtual phase calibrating model planes, J. Opt. A: Pure Appl. Opt. 7 (4) (2005) 192-197.

[115] L. Zhongwei, S. Yusheng, W. Congjun, W. Yuanyuan, Accurate calibration method for a structured light system, Opt. Eng. 47 (5) (2008) 053604.

[116] R. Anchini, G. Di Leo, C. Liguori, A. Paolillo, A new calibration procedure for 3-D shape measurement system based on phase-shifting projected fringe profilometry, IEEE Trans. Instrumentation and Measurement 58 (5) (2009) 1291-1298.

[117] E. Zappa, G. Busca, Fourier-transform profilometry calibration based on an exhaustive geometric model of the system, Opt. Laser Eng. 47 (7-8) (2009) 754-767.

[118] X. Chen, J. Xi, Y. Jin, J. Sun, Accurate calibration for a camera-projector measurement system based on structured light projection, Opt. Laser Eng. 47 (3-4) (2009) 310-319.

[119] Z. Wang, H. Du, H. Bi, Out-of-plane shape determination in generalized fringe projection profilometry, Opt. Express 14 (25) (2006) 1212212133

[120] H. Du, Z. Wang, Three-dimensional shape measurement with an arbitrarily arranged fringe projection profilometry system, Opt. Lett. 32 (16) (2007) 2438-2440.
[121] X. Mao, W. Chen, X. Su, Improved Fourier-transform profilometry, Appl. Opt. 46 (5) (2007) 664-668.

[122] C. Yu, Q. Peng, A unified-calibration method in FTP-based 3D data acquisition for reverse engineering, Opt. Laser Eng. 45 (3) (2007) 396404.

[123] D. Feipeng, G. Shaoyan, Flexible three-dimensional measurement technique based on a digital light processing projector, Appl. Opt. 47 (3) (2008) 377-385.

[124] W. H. Wang, Y. S. Wong, G. S. Hong, 3D measurement of crater wear by phase shifting method, Wear 261 (2) (2006) 164-171.

[125] C. Zhang, P. S. Huang, F. Chiang, Microscopic phase-shifting profilometry based on digital micromirror device technology, Appl. Opt. 41 (28) (2002) 5896-5904.

[126] C. Quan, X. Y. He, C. F. Wang, C. J. Tay, H. M. Shang, Shape measurement of small objects using LCD fringe projection with phase shifting, Opt. Commun. 189 (1-3) (2001) 21-29.

[127] W. Jia, H. Qiu, A novel optical method in micro drop deformation measurements, Opt. Laser Eng. 35 (3) (2001) 187-198.

[128] W. Li, X. Su, Z. Liu, Large-scale three-dimensional object measurement: A practical coordinate mapping and image data-patching method, Appl. Opt. 40 (20) (2001) 3326-3333.

[129] M. Heredia-Ortiz, E. A. Patterson, On the industrial applications of Moiré and fringe projection techniques, Strain 39 (3) (2003) 95-100.

[130] S. Pavageau, R. Dallier, N. Servagent, T. Bosch, A new algorithm for large surfaces profiling by fringe projection, Sensors and Actuators A: Physical 115 (2-3) (2004) 178-184.

[131] Y. Li, J. A. Nemes, A. Derdouri, Optical 3-D dynamic measurement system and its application to polymer membrane inflation tests, Opt. Laser Eng. 33 (4) (2000) 261-276.

[132] X. Su, W. Chen, Q. Zhang, Y. Chao, Dynamic 3-D shape measurement method based on FTP, Opt. Laser Eng. 36 (1) (2001) 49-64.

[133] W. Van Paepegem, A. Shulev, A. Moentjens, J. Harizanova, J. Degrieck, V. Sainov, Use of projection moiré for measuring the instantaneous outof-plane deflections of composite plates subject to bird strike, Opt. Laser Eng. 46 (7) (2008) 527-534.

[134] E. Hu, Y. He, Surface profile measurement of moving objects by using an improved pi phase-shifting Fourier transform profilometry, Opt. Laser Eng. 47 (1) (2009) 57-61.

[135] J. Vanherzeele, S. Vanlanduit, P. Guillaume, Processing optical measurements using a regressive Fourier series: A review, Opt. Laser Eng. 47 (34) (2009) 461-472.

[136] Q. Kemao, Two-dimensional windowed Fourier transform for fringe pattern analysis: Principles, applications and implementations, Opt. Laser Eng. 45 (2) (2007) 304-317.

[137] T. Yan, C. Wen-Jing, Z. Qiang, S. Xian-Yu, X. Li-Qun, BP neural network applied to 3D object measurement based on fringe pattern projection, Optik 120 (7) (2009) 347-350.

[138] J. Villa, M. Servin, L. Castillo, Profilometry for the measurement of 3D object shapes based on regularized filters, Opt. Commun. 161 (1-3) (1999) 13-18.

[139] F. Berryman, P. Pynsent, J. Cubillo, A theoretical comparison of three fringe analysis methods for determining the three-dimensional shape of an object in the presence of noise, Opt. Laser Eng. 39 (1) (2003) 35-50.

[140] R. M. Goldstein, H. A. Zebker, C. L. Werner, Satellite radar interferometry: two-dimensional phase unwrapping, Radio Science 23 (4) (1988) 713-720.

[141] J. M. B. Dias, J. M. N. Leitao, The ZpiM algorithm: A method for interferometric image reconstruction in SAR/SAS, IEEE Trans. Image Processing 11 (4) (2002) 408-422.

142] B. Jose, V. Katkovnik, J. Astola, K. Egiazarian, Absolute phase estimation: adaptive local denoising and global unwrapping, Appl. Opt. 47 (29) (2008) 5358-5369.

[143] T. R. Judge, P. J. Bryanston-Cross, A review of phase unwrapping techniques in fringe analysis, Opt. Laser Eng. 21 (4) (1994) 199-239.

[144] J. M. Huntley, C. R. Coggrave, Progress in phase unwrapping, Proc. SPIE 3407 (1998) 86-93.

[145] D. C. Ghiglia, M. D. Pritt, Two-Dimensional Phase Unwrapping: Theory, Algorithms, and Software, Wiley-Interscience, 1998.

[146] X. Su, W. Chen, Reliability-guided phase unwrapping algorithm: A review, Opt. Laser Eng. 42 (3) (2004) 245-261.

[147] E. Zappa, G. Busca, Comparison of eight unwrapping algorithms ap- 
plied to Fourier-transform profilometry, Opt. Laser Eng. 46 (2) (2008) $106-116$.

[148] H. Guo, M. Chen, P. Zheng, Least-squares fitting of carrier phase distribution by using a rational function in profilometry fringe projection, Opt. Lett. 31 (24) (2006) 3588-3590.

[149] C. Quan, C. J. Tay, L. J. Chen, A study on carrier-removal techniques in fringe projection profilometry, Opt. Laser Techn. 39 (6) (2007) 11551161.

[150] J. Salvi, X. Armangue, J. Batlle, A comparative review of camera calibrating methods with accuracy evaluation, Pattern Recognition 35 (7) (2002) 1617-1635.

[151] S. Zhang, P. S. Huang, Novel method for structured light system calibration, Opt. Eng. 45 (8) (2006) 083601.

[152] J. Y. Bouguet, Camera calibration toolbox for MATLAB. www.vision.caltech.edu/bouguet $j /$ calib_doc.

[153] P. Jia, J. Kofman, C. English, Comparison of linear and nonlinear calibration methods for phase-measuring profilometry, Opt. Eng. 46 (4) (2007) 043601.

[154] A. S. Georghiades, P. N. Belhumeur, D. J. Kriegman, From few to many: Illumination cone models for face recognition under variable lighting and pose, IEEE Trans. Pattern Analysis and Machine Intelligence 23 (6) (2001) 643-660.

[155] Y. Hao, Y. Zhao, D. Li, Shape measurement of objects with large discontinuities and surface isolations using complementary grating projection, Proc. of SPIE 3898 (1999) 338-343.

[156] O. A. Skydan, M. J. Lalor, D. R. Burton, Technique for phase measurement and surface reconstruction by use of colored structured light, Appl. Opt. 41 (29) (2002) 6104-6117.

[157] J. Harizanova, V. Sainov, Three-dimensional profilometry by symmetrical fringes projection technique, Opt. Laser Eng. 44 (12) (2006) 12701282.

[158] M. Sasso, G. Chiappini, G. Palmieri, D. Amodio, Superimposed fringe projection for three-dimensional shape acquisition by image analysis, Appl. Opt. 48 (13) (2009) 2410-2420.

[159] Y. Cai, X. Su, Inverse projected-fringe technique based on multi projectors, Opt. Laser Eng. 45 (10) (2007) 1028-1034.

[160] M. J. Baker, J. F. Chicharo, J. Xi, An investigation into temporal gamma luminance for digital fringe fourier transform profilometers, IEEE Int. Symp. Intelligent Signal Proc. (4447501) (2007).

[161] B. Pan, Q. Kemao, L. Huang, A. Asundi, Phase error analysis and compensation for nonsinusoidal waveforms in phase-shifting digital fringe projection profilometry, Opt. Lett. 34 (4) (2009) 416-418.

[162] Z. Li, Y. Shi, C. Wang, D. Qin, K. Huang, Complex object 3D measurement based on phase-shifting and a neural network, Opt. Commun. 282 (14) (2009) 2699-2706.

[163] S. Zhang, S. Yau, Generic nonsinusoidal phase error correction for threedimensional shape measurement using a digital video projector, Appl. Opt. 46 (1) (2007) 36-43.

[164] L. Xiong, S. Jia, Phase-error analysis and elimination for nonsinusoidal waveforms in Hilbert transform digital-fringe projection profilometry, Opt. Lett. 34 (15) (2009) 2363-2365.

[165] S. Zhang, P. S. Huang, High-resolution, real-time three-dimensional shape measurement, Opt. Eng. 45 (12) (2006) 123601.

[166] P. S. Huang, C. Zhang, F. Chiang, High-speed 3-D shape measurement based on digital fringe projection, Opt. Eng. 42 (1) (2003) 163-168.

[167] Z. Zhang, C. E. Towers, D. P. Towers, Time efficient color fringe projection system for 3D shape and color using optimum 3-frequency selection, Opt. Express 14 (14) (2006) 6444-6455.

[168] L. Kinell, Multichannel method for absolute shape measurement using projected fringes, Opt. Laser Eng. 41 (1) (2004) 57-71.

[169] Y. Hu, J. Xi, E. Li, J. Chicharo, Z. Yang, Y. Yu, A calibration approach for decoupling colour cross-talk using nonlinear blind signal separation network, Proc. of IEEE Conference on Optoelectronic and Microelectronic Materials and Devices (1577541) (2005) 265-268.

[170] Y. Hu, J. Xi, J. Chicharo, Z. Yang, Blind color isolation for colorchannel-based fringe pattern profilometry using digital projection, J. Opt. Soc. Am. A 24 (8) (2007) 2372-2382.

[171] H. Zhao, W. Chen, Y. Tan, Phase-unwrapping algorithm for the measurement of three-dimensional object shapes, Appl. Opt. 33 (20) (1994) $4497-4500$.

[172] J. Li, L. G. Hassebrook, C. Guan, Optimized two-frequency phase- measuring-profilometry light-sensor temporal-noise sensitivity, J. Opt. Soc. Am. A 20 (1) (2003) 106-115.

[173] L. Kinell, M. Sjodahl, Robustness of reduced temporal phase unwrapping in the measurement of shape, Appl. Opt. 40 (14) (2001) 2297-2303.

[174] H. Zhang, M. J. Lalor, D. R. Burton, Spatiotemporal phase unwrapping for the measurement of discontinuous objects in dynamic fringeprojection phase-shifting profilometry, Appl. Opt. 38 (16) (1999) 3534 3541.

[175] J. M. Huntley, H. O. Saldner, Error-reduction methods for shape measurement by temporal phase unwrapping, J. Opt. Soc. Am. A 14 (12) (1997) 3188-3196.

[176] J. Tian, X. Peng, X. Zhao, A generalized temporal phase unwrapping algorithm for three-dimensional profilometry, Opt. Laser Eng. 46 (4) (2008) 336-342.

[177] W. Liu, Z. Wang, G. Mu, Z. Fang, Color-coded projection grating method for shape measurement with a single exposure, Appl. Opt. 39 (20) (2000) 3504-3508.

[178] J. Pages, J. Salvi, C. Collewet, J. Forest, Optimised de Bruijn patterns for one-shot shape acquisition, vol. 23, 707-720, 2005.

[179] P. Fong, F. Buron, Sensing deforming and moving objects with commercial off the shelf hardware, in: Proc. of the IEEE Computer Society Conference on Computer Vision and Pattern Recognition, vol. 3, 101, 2005.

[180] S. S. Gorthi, K. R. Lolla, Novel single-shot structured light technique for accurate, reliable and dense 3D shape measurement, in: Proc. of the Int. Conference on Optics and Optoelectronics, OP-OIP-6, 2005.

[181] W. Su, Color-encoded fringe projection for 3D shape measurements, Opt. Express 15 (20) (2007) 13167-13181.

[182] H. J. Chen, J. Zhang, D. J. Lv, J. Fang, 3-D shape measurement by composite pattern projection and hybrid processing, Opt. Express 15 (19) (2007) 12318-12330.

[183] W. Su, Projected fringe profilometry using the area-encoded algorithm for spatially isolated and dynamic objects, Opt. Express 16 (4) (2008) 2590-2596.

[184] M. Takeda, Q. Gu, M. Kinoshita, H. Takai, Y. Takahashi, Frequency-multiplex Fourier-transform profilometry: A single-shot three-dimensional shape measurement of objects with large height discontinuities and/or surface isolations, Appl. Opt. 36 (22) (1997) 53475354.

[185] J. Zhong, Y. Zhang, Absolute phase-measurement technique based on number theory in multifrequency grating projection profilometry, Appl. Opt. 40 (4) (2001) 492-500.

[186] D. Choudhury, M. Takeda, Frequency-multiplexed profilometric phase coding for three-dimensional object recognition without 2pi phase ambiguity, Opt. Letters 27 (16) (2002) 1466-1468.

[187] C. Guan, L. G. Hassebrook, D. L. Lau, Composite structured light pattern for three-dimensional video, Opt. Express 11 (5) (2003) 406-417.

[188] W. Su, H. Liu, Calibration-based two-frequency projected fringe profilometry: A robust, accurate, and single-shot measurement for objects with large depth discontinuities, Opt. Express 14 (20) (2006) 91789187.

Sai Siva Gorthi

Pramod Rastogi

Applied Computing and Mechanics Laboratory, Ecole Polytechnique Fédérale de Lausanne, 1015, Switzerland E-mail address: pramod.rastogi@epfl.ch 